\title{
FAKE INDIAN CURRENCY DETECTION USING DEEP LEARNING
}

\author{
Juliet D'cruz \\ Computer Science and Engineering \\ Mar Baselios Institute of Technology and Science \\ Kothamangalam, India \\ Mariya Jose \\ Computer Science and Engineering \\ Mar Baselios Institute of Technology and Science \\ Kothamangalam, India
}

\begin{abstract}
Indian rupee is the official currency of India. Issuance of Indian currency is controlled by the Reserve Bank of India. In real time, people could only authenticate money, because the observation ability of the human eyes are limited, and they are difficult to distinguish the fake and real currency without the technology. In this paper a new approach for the identification of fake currency notes is explained. The method is based on Deep Learning, which has seen a great success in image classification tasks. An artificial data-set was generated by using the different image sets to assist training and testing of the deep convolutional neural network (DCNN) model. Under the availability of a data-set, the deep neural networks can be better trained. We are testing the features like serial number, security strip, face of gandhi, dot pattern, translucent of gandhi, reserve bank emblem. Such a model may then be built into a smart-phone android app and can help people in detecting fake notes in case of suspicion in real time with just an image taken through the smart-phones camera.
\end{abstract}

Keywords-Deep learning

\section{INTRODUCTION}

This identification of fake currency is very important in many sectors like banking, economy and so on. This system here proposed is used to detect whether the given currency is counterfeit or not through a convolution neural network. In this approach, Deep learning used for the recognition and classification of images from a large data sets, which is used in object recognition. A deep neural network is a better model that works in a similar way to the neurons in the human brain. In this model, each neuron takes an input, performs some

\author{
Mariya Eldhose \\ Computer Science and Engineering \\ Mar Baselios Institute of Technology and Science \\ Kothamangalam, India \\ Bonia Jose \\ Computer Science and Engineering \\ Mar Baselios Institute of Technology and Science \\ Kothamangalam, India
}

operations then it passes it's output to the next neuron that is to its hidden layer.

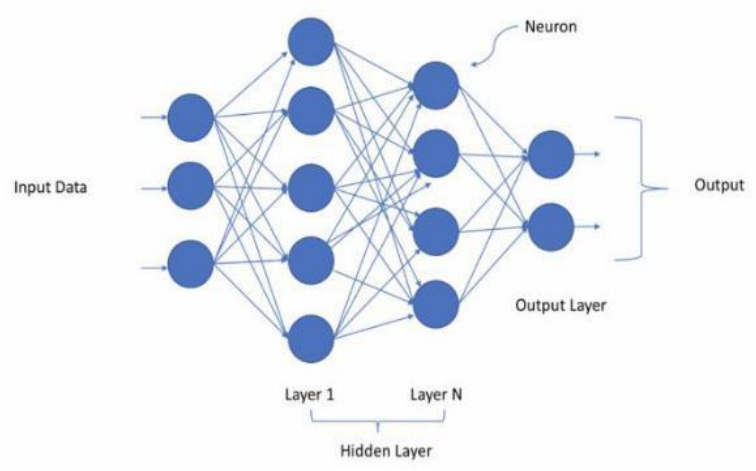

Fig. Deep Neural Network

II. EXISTING METHODS

A. Look Through Register: There is a small floral design is printed through middle portion of the vertical band and very next to watermark. The floral design on the front portion is hollow and it's back side is filled up clearly. The design will see as a floral design when it seen against the light[1].

B. Fluorescence: The Fluorescent ink is used to print number panels on the notes. The currencies also contains an optical fiber. When the currency is exposed to light, can see the number panel in fluorescent ink and the optical fiber in the note[1].

C. Optically Variable Ink: This is used as a security feature. This feature found in the Rs.200, 500, and Rs. 2000 bank notes. In Nov. 2000 Optically variable ink as security feature for bank note is issued. The denomination value is printed in the note with the help of an optical variable ink. When the note is flat, the 
color of numerical 2000 or 500 appear as green and a change occurred the color to blue when the note held in a particular angle[1].

D. Water marking: One of the important feature in Currency. The Mahatma Gandhi watermark is present in every bank notes. The watermark is present with a shade effect and there are multidirectional lines in the watermark[1].

E. Security Thread: Security thread in the 2000 and 500 note, it appears on the left side of the Mahatma Gandhi's portrait. In security thread, the visible features are - RBI and -BHARAT. When the currency is held against the light, security thread can be seen as a continuous line[1].

F. Identification Mark: Every note has its own special identification mark. There exists different shapes of identification mark for different currency (Rs.500circle and Rs.2000- Square). The identification mark present on the left side of the water mark[1].

G. Latent Image: Here the latent image shows the denomination value in numerical. On the observe side of notes, the latent image is present on the right of Mahatma Gandhi portrait as vertical band. The image is visible when the note is held horizontally at eye[1].

H. Micro Lettering: The micro letter's appears in just between the Mahatma Gandhi portrait and vertical band. It contains the denomination value of bank note in micro letters. The denomination value can be seen under magnifying glass[1].

\section{DEEP LEARNING}

Deep learning is a neural network includes multiple layers that is used to learn multiple images as the dataset. It has been mainly used in the fields of vision, image recognition, object detection and speech. Deep learning is used to estimate the weights in each layer through the back propagation algorithm.

The effect of processing in different layers is different and also the process is much complicated. In artificial neural networks, supervised learning and unsupervised learning are all associated very closely. In 2015, a neural network system based on Max- Pooling Convolution has been proposed, which could be used for image recognition, object detection is classified according to different datasets.

Through the deep learning, each neuron in the layer could be activated, but the complexity will be increased with the increase in the levels go up. The performance of deep learning is much better at solving practical problems when compared to traditional machine learning methods. The levels of complexity and layers in deep learning are different for different data, but with more data, the more layers of a neural network, the simpler it becomes, because of this reasons; deep learning has become a main streaming technology in computing.
Although the applications of deep learning are much more wider than that of traditional machine learning [2].

\subsection{Architecture of CNN}

$\mathrm{CNN}$ is a back propagating neural network with a weight kernel of 2 Dimensional. For our experiment we have used the python libraries like Theano and TensorFlow for implementing and training the deep learning model. There are many pre-trained models available on the internet like Le Net, VGG Net, Alex Net, ZF Net, Google Net/Inception, ResNet and SSD Model. Here we use SSD Model [3].

\subsection{Single Shot Multi Box Detector}

Single Shot Multi Box Detector (SSD) is a frame model, which is used for object detection. This model generates bounding boxes from feature maps on different layers, the outcome of these bounding boxes is obtained. It will form different bounding boxes according to different classifiers after the classification and we could determine what the object it is. It is also used in real-time detection, but it is faster than R-CNN and ResNets. However, SSD guarantees the speed of detection and also ensures the accuracy of detection [2].

\section{THEORY AND EXPERIMENTATION}

To carry out the proposed solution of finding false currency, we perform the operations using $\mathrm{CNN}$ with the help of python language.

\subsection{Proposed Solution}

To build a convolutional neural network according to proposed algorithm which will be trained on the given fake and original currency data set, and later be able to predict whether the given currency image is fake or original. The way we are going to achieve it is by training an artificial neural network on image data set of currency and make the NN (Neural Network) to predict which class the image belongs to, when it sees an image having fake note or original note the next time. Convolutional neural networks (CNN's) are nowadays widely used in pattern-recognition and image-recognition problems. They have many advantages compared to other techniques. Typically, Convolution neural networks use approximately 5 to 25 distinct layers of pattern recognition. They take raw data, without the need for an initial separate pre-processing or feature extraction stage: in a $\mathrm{CNN}$, the feature extraction and classification occur naturally within a single framework. This is a major advantage when compared to other image processing techniques. 
44.1.1. Steps for currency detection

1. Input the Image: Take the image of the note that want to check the originality. This is done by using the Camera of the phone.

2.Feature Extraction: Here, the different features of the currency id determined. Features like Serial Number from left end and right end, Security strip, Side strips, Face of Gandhiji, Image of Reserve Bank emblem and so on. In this method, these features from each denomination is used to train the model to detect the counterfeit currencies.

3.Training the Data: Collect the labelled images. Convert $\mathrm{xml}$ files of the images into a csv file and then into record. After the conversion put all the resources together into a resource bucket for the training of the model. Then the trained data is used to make the comparisons to detect the given image of currency is fake or not.

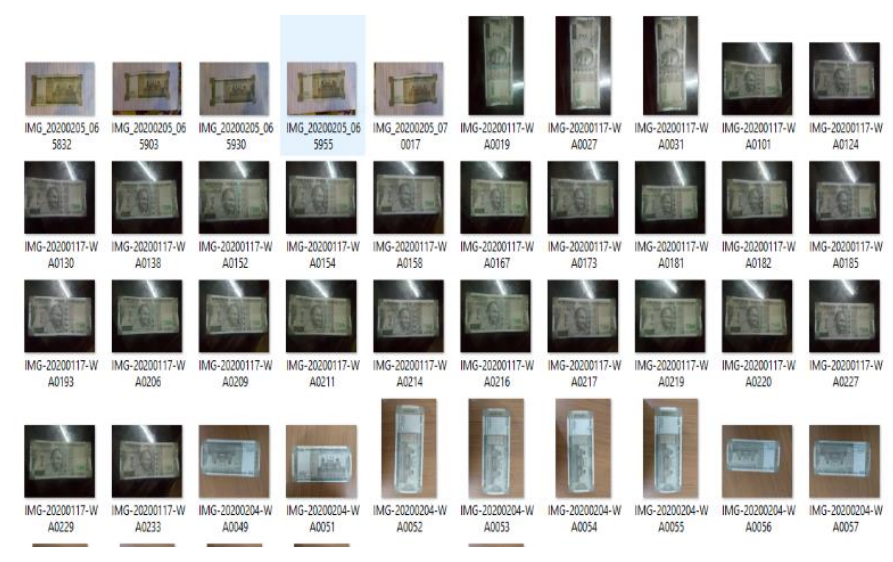

Fig.4.1.1.1 Data Set of the currency

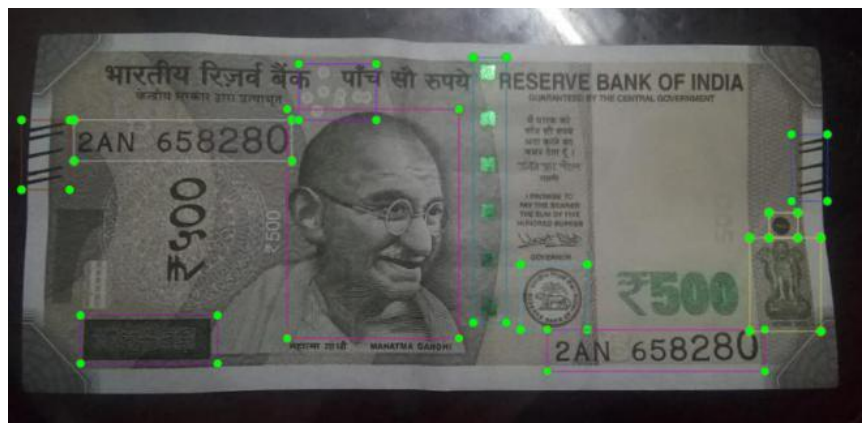

Fig.4.1.1.2 Labelled real Currency

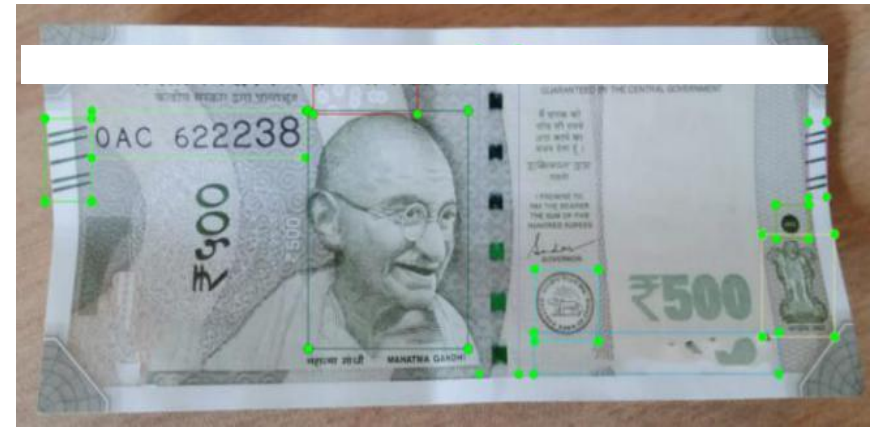

Fig.4.1.1.3 Labelled fake Currency

\section{METHODOLOGY}

\subsection{Research Design}

Main content of our project is currency recognition. Before we start the research, we need to make a design of the project. Before we formally start our project, we need some basic ideas for the specific content of each process. As shown in Fig. 1, this flowchart shows the basic concept of the process taken place. In currency recognition data set collection is the main part, we need the pictures of both front side and backside. Before training the data, the data set needs to be labeled, and need to be resize the dataset as per requirements After fulfilled the work, we send them to the convolutional layers for currency classification. SSD's architecture builds on the venerable VGG-16 architecture, but discards the fully connected layers, due to its complexity. The reason VGG-16 was used as the base network is because of its strong performance in high quality image classification tasks and its popularity for problems where transfer learning helps in improving results. Instead of the original VGG fully connected layers, a set of auxiliary convolutional layers were added, thus enabling to extract features at multiple scales and progressively decrease the size of the input to each subsequent layer.

\subsection{Data Collection}

In our research we want to find the counterfiet currency in 500 and 2000 rupee notes. Each currency of each denomination will have front and back sides. In real time we can capture the photo of the currency. During the image taking process, we need to make sure that the currency is flat, and the currency could be fully displayed in the image. Simultaneously, we take the photos of the front and back side of the currency. We also need to ensure that when we are taking the photo, there is enough light around it, because we expect to capture the details of currency more clearly. After making the dataset it is converted to CSV file and record. And we need to edit the image to ensure that the 
currency is clear and complete in each picture, there is not any parts out of the boxes. In this case, we get 100 pictures of front image and 96 pictures of back image, we have obtained a total of 196 pictures as a dataset, the resolution of each picture is $300 \times 300$ and $512 \times 512$.

\subsection{Data Augmentation}

After the data collection, we have carried out the procedure of data manual marking; we grouped all the collected data into six categories, and we obtained a total of 250 valid currency raw images for the purpose of recognition. But this number of samples is insufficient, it is difficult to support the training of deep learning. In SSD it is stated that data augmentation, like in many other deep learning applications, has been crucial to teach the network to become more robust to various object sizes in the input. To this end, they generated additional training examples with patches of the original image at different IoU ratios and random patches as well. Moreover, each image is also randomly horizontally flipped with a probability of 0.5 , thereby making sure potential objects appear on left and right with similar likelihood. After the data augmentation, each of the original pictures can obtain 25 currency enhanced images. In other words, our original 300 raw images will receive 30025 images in the dataset after the argumentation; the number of datasets will be expanded from the previous 250 to 6000 , thus it greatly improves the efficiency and integrity. The dataset after data argumentation can make our research experiments more precise. In this way, we can increase the position, and adjust the color of the currency image.

\subsection{CNN MODEL}

As the data training progresses, the training layer continually updates the parameters of each layer, so we can see the connection of the training layer. At the same time, each layer uses a random initial parameter value and connects to the initial layer, we save the updated parameters to the save layer on the hard disk. Finally, the obtained data is input to the valid layer, and it is verified with the trained dataset to determine whether the dataset is fully trained. In this paper, currency detection, as a subtask of currency identification, needs to be divided into two parts in training; that is, positioning and classification in MLP, the overall learning framework is TensorFlow. Specifically, we split it into convolutional layers and fully connected layers.

\section{Limitations}

For currency recognition, using $\mathrm{CNN}$ as the feature extractor, SSD is the basic model of the overall framework, and $80 \%$ of the time is spent on the base VGG-16 network; this means that with a faster and equally accurate network
SSD's performance could be even better but there are still some limitations.

The inability to recover old data and solid state drives are highly expensive and are sold with a hefty price tag unlike conventional HDDs are the biggest disadvantage of it.

\section{CONCLUSION}

The main purpose of this paper is to find the counterfeit currency. We trained the SSD framework. These models are based on empirical methods. We have also received satisfactory results. We use VGG-16 $\mathrm{CNN}$ as a feature extractor under the framework of Single Shot Multi Box Detector (SSD) model. The model achieves $92.7 \%$ top-5 test accuracy in ImageNet, which is a dataset of over 14 million images belonging to 1000 classes. SSD Generate bounding boxes from feature maps on different layers. High speed and accuracy. It is trained using Tensorflow. We are testing the features like Security Thread, UV Lines, RBI Logo, Identification Mark, Face of Gandhiji, Serial Number and stripes. The main contribution is through using CNN and SSD, with an accuracy of $90.6 \% 2$.

\section{ACKNOWLEDGEMENT}

I would like to convey my heartfelt gratitude towards my guide, Mrs. Bonia Jose for her constant guidance, encouraging help and inspiring words. I am thankful to the department of Computer Science and Engineering for their support.

\section{REFERENCE}

[1] Qian Zhang,Wei Qi Yan(2018). Currency Detection and Recognition Based on Deep Learning, DOI: 10.1109/AVSS.2018.8639124.

[2] M. Laavanya and V. Vijayaraghavan (2019) . Real Time Fake Currency Note Detection Using Deep Learning, DOI: 10.35940/ijeat.a1007.1291s52019.

[3] Tushar Agasti, Gajanan Burand, Pratik Wade and P chitra (2017). Fake Currency Detection Using Image Processing DOI: 10.1088

[4] Arya, s, Dr. M. Sasikumar(2019); Fake Currency Detection DOI: 10.1109/ICRAECC4387.2019.8994968

[5]. Vivek Sharan, Amandeep Kaur(2019) .Detection of Counterfiet Indian Currency Note Using Image Processing, DOI: 10.1109/i2ct45611.2019.9033740 
[6] Qian Zhang, Wei QI Yan(2018). Currency Detection and Recognition Based on Deep Learning.DOI:10.1109/AVSS 2018.8639124

[7]. Kiran Kamble, Anuthi Bhansali, Pranali Satalgaonkar,

Shruti Alagundgi (2019). Counterfeit Currency Detection Using Deep Convolutional Network, DOI: 10.1109/PuneCon46936.2019.9105683

[8]. Qian Zhang, Wei Qi Yan and Mohan Kankanhalli (2019). Overview of Currency RecognitionUsing Deep Learning, DOI: $\quad$ 10.1007/s42786-018-00007-

[9]. Raj Shah, Mayank Champaneri, Pujan Sheth, Vaishali

Gaikwad (2018). Currency Counting Fake Note Detection, DOI: 10.2139/ssrn.3275068.

[10]. Snehlata, Vipin Saxena (2019). An Efficient Technique for Detection of Fake

Currency, DOI: 10.35940/ijrte.B3196.098319

[11]. Prof. S.R.Javheri, Shitalpadole, PoojaDeshmukh, ShitalKhade (2016). Mobile based application for fake currency detection and recognition, DOI: 10.17148/IJARCCE.2016.5536

[12]. S. A. Bhavani (2017). Currency Recognition Using SIFT, DOI: 10.5120/ijca2017914368

[13]. Yepuri Spoorthi Hawanika, Kapavarapu Sai Sreeja, Yangala Shamitha, Bandaru Yuva Priyanka, Manam Srilatha (2020). Indian Counterfeit Currency Detection, DOI: 10.35940/ijitee.F4716.0496420

[14]. Mr. Prasad D Deshpande, Prof Atul Shrivastava (2018). Security Features Analysis of Indian Currency for Recognition and Authentication, DOI: 10.22214/ijraset.2018.3120

[15]. Ashish Nagar, Dr. Manish Sharma, Sohit Agarwal(2019) Fake Identification by Android Mobile Phone Using Digital Image Processing DOI: 10.22214//ijraset.2019 
International Journal of Engineering Applied Sciences and Technology, 2020

Vol. 5, Issue 1, ISSN No. 2455-2143, Pages 720-724

Published Online May 2020 in IJEAST (http://www.ijeast.com) 